\title{
Borel Summability of the Unequal Double Well
}

\author{
S. Graffi ${ }^{1}$ and V. Grecchi ${ }^{2}$ \\ 1 Dipartimento di Matematica, Università di Bologna, I-40126 Bologna, Italy \\ 2 Istituto di Matematica, Università di Modena, I-41100 Modena, Italy
}

\begin{abstract}
Unlike the $\varepsilon=0$ case, the perturbation series of the unequal double well $p^{2}+x^{2}+2 g x^{3}+g^{2}(1+\varepsilon) x^{4}$ are Borel summable to the eigenvalues for any $\varepsilon>0$.
\end{abstract}

The best known example (see e.g. [13, Sect. XII.4]) of a non-Borel summable perturbation series is represented by the Rayleigh-Schrödinger perturbation expansion (hereafter RSPE) of the standard double well oscillator $H(g)=p^{2}+x^{2}$ $+x^{2}(1+g x)^{2}$ in $L^{2}(\mathbb{R}), g \in \mathbb{R}$. This fact is of course due to the instability of the eigenvalues as $g \rightarrow 0$, i.e. to their asymptotic degeneracy as $g \rightarrow 0$. However there are examples, such as the Herbst and Simon [5] one, $K(g)=p^{2}+x^{2}(1+g x)^{2}-2 g x-1$, in which there is stability but no Borel summability to the eigenvalues. Hence, also on account of recent investigations on Borel summability in four dimensional field theories $[6,7]$, it could be interesting to relate the lack of summability to some other more subtle physical mechanism of well defined meaning also in a more general context. To this end, T. Spencer has suggested considering the following "unequal" double well oscillator

$$
H(g, \varepsilon)=p^{2}+x^{2}(1+g x)^{2}+\varepsilon g^{2} x^{4},
$$

which in the limit $g \rightarrow 0$ has an infinite action instanton for any $\varepsilon \geqq 0$. (A standard reference for the notion of instanton in problems of this type is Coleman [1]; additional discussion can be found in $[2,11]$.) This model could in addition have some interest in itself: as a matter of fact, in some sense it represents the slightest modification of the non-summable example, and it is natural to ask to what extent the non-summability as "accidental," i.e. how sensitive is its dependence on the choice of the parameters in $H(g)$ ? Furthermore it can be easily proved through the Hunziker-Vock technique [8] that any eigenvalue $E$ of $H(0, \varepsilon) \equiv H(0)=p^{2}+x^{2}$ is stable for $g \in \mathbb{R}$ small as an eigenvalue of $H(g, \varepsilon), \varepsilon>0$, because the second minimum of $V(g, \varepsilon) \equiv x^{2}(1+g x)^{2}+\varepsilon g^{2} x^{4}$ tends to $+\infty$ as $g \rightarrow 0, \varepsilon>0$. 
In this note we prove that, for $\varepsilon>0$, any eigenvalue $E$ is actually stable as an eigenvalue $E(g, \varepsilon)$ of $H(g, \varepsilon)$ for $g$ complex, $|g|$ suitably small, $|\arg g| \leqq \frac{\pi}{4}$, and that the RSPE near $E$ is Borel summable to $E(g, \varepsilon)$ for $g$ positive and small. To this end, let us first collect some well known results on the operator families $H(g, \varepsilon)$ acting in $L^{2}(\mathbb{R})$ under the form of a proposition whose proof can be easily traced out of $[13$, Sect. XII.3, 4].

Proposition 1. Let $\varepsilon>0$ be fixed and $g \in \mathbb{C}, g=|g| e^{i \theta},|g| \geqq 0,|\theta|<\frac{\pi}{2}$. Let the operator family $H(g, \varepsilon)$ in $L^{2}(\mathbb{R})$ be defined as the action of $p^{2}+V(g, \varepsilon)$ on the domain $D(H(g, \varepsilon))=D\left(p^{2}\right) \cap D\left(x^{4}\right), g \neq 0$, and $H(0)$ as the action of $p^{2}+x^{2}$ on $D(0)$ $=D\left(p^{2}\right) \cap D\left(x^{2}\right)$ for $g=0$. Then for any fixed $g, H(g, \varepsilon)$ has compact resolvent, and any eigenvalue $E(g, \varepsilon)$ is a locally holomorphic function of $g$ in the complex sector

$$
S \equiv\left\{g \in \mathbb{C}:|g|>0,|\theta|<\frac{\pi}{2}\right\} .
$$

Our result can be stated as follows.

Proposition 2. Let $\varepsilon>0$ be fixed. Then:

(i) There is $B(E)>0$ such that any eigenvalue $E$ of $H(0)$ is stable (in the sense of Kato [9, Sect. VIII.1.4]) as an eigenvalue $E(g, \varepsilon)$ of $H(g, \varepsilon)$ for $|g|<B(E),|\theta| \leqq \frac{\pi}{4}$.

(ii) $E(g, \varepsilon)$ is a holomorphic function of $g$ at least in the sector

$$
Q \equiv\left\{0<|g|<B(E) ;|\theta| \leqq \frac{\pi}{4}\right\}
$$

and is continuous as $|g| \rightarrow 0,|\theta| \leqq \frac{\pi}{4}$.

(iii) Let $\sum_{n=0}^{\infty} A_{n}(\varepsilon) g^{n} \sim E(g, \varepsilon)$ be the RSPE of $E(g, \varepsilon)$ near $E, R_{N}(g, \varepsilon)=E(g, \varepsilon)$ $-\sum_{n=0}^{N-1} A_{n}(\varepsilon) g^{n}$ its $N^{\text {th }}$ order remainder. Then $A_{2 n+1}(\varepsilon) \equiv 0 \forall n$, and there is $D>0$ independent of $g$ such that

$$
\left|R_{2 N}(g, \varepsilon)\right| \leqq D N !\left|g^{2}\right|^{N}, \quad N=1,2, \ldots
$$

as long as $|g| \leqq B(E),|\theta| \leqq \frac{\pi}{4}$.

Remarks. (i) Statement (iii) implies the Borel summability of $\sum_{n=0}^{\infty} A_{n}(\varepsilon) g^{n}$ to $E(g, \varepsilon)$ for $0 \leqq g \leqq B(E)$. For $A_{2 n+1} \equiv 0 \forall n$ implies that $E(g, \varepsilon)$ is a function of $g^{2}$, which by (ii) is holomorphic for $0<|g|^{2}<B(E)^{2},\left|\arg g^{2}\right| \leqq \frac{\pi}{2}$, and continuous as $|g| \rightarrow 0$, $\left|\arg g^{2}\right| \leqq \frac{\pi}{2}$. Then (2) holds with $g$ replaced by $g^{2}$ in the left-hand side so that by the Watson-Nevanlinna theorem (for details see Sokal [14]) the summability takes place for $g$ as above.

(ii) Proposition 1 allows us to apply the standard complex scaling argument (see e.g. Simon [12] for details). Hence the operators $H(g . \varepsilon)$ and $e^{-2 i \phi} H(g, \varepsilon, \phi)$, 
$H(g, \varepsilon, \phi)$ defined as the action of $p^{2}+e^{4 i \phi} V\left(g e^{i \phi}, \varepsilon\right)$ on $D(H(g, \varepsilon))$ have the same eigenvalues as long as $|\theta+3 \phi|<\frac{\pi}{2}$.

Let us now proceed to prove Proposition 2. By Remark (ii) we can consider $H(g, \varepsilon, \phi)$ instead of $H(g, \varepsilon)$. Given $\delta, 0<\delta<\frac{\pi}{4}$, we take $\phi=-\theta \equiv-\arg g$ for $|\theta| \leqq \frac{\pi}{4}-\delta$, i.e. we consider $H(|g|, \varepsilon,-\theta)$ as long as $|\theta| \leqq \frac{\pi}{4}-\delta$. For $\theta=\frac{\pi}{4}-\theta^{\prime}$, $0 \leqq \theta^{\prime} \leqq \delta_{1}, \delta<\delta_{1}<\frac{\pi}{4}$, we take $\phi=-\frac{\pi}{4}+\eta, \delta_{1}<\eta<\frac{\pi}{4}, \eta<\operatorname{arctg} \sqrt{\varepsilon}$, i.e. we consider $H(|g|, \varepsilon, \chi) \equiv p^{2}+e^{-i(\pi-4 \eta)} V\left(|g| e^{i \chi}, \varepsilon\right)$ with $\chi=\eta-\theta^{\prime}, \delta_{1}-\delta \leqq \chi \leqq \eta$. For $\theta=-\frac{\pi}{4}+\theta^{\prime}$ we obviously consider $H(|g|, \varepsilon,-\chi)$. The condition $\eta<\operatorname{arctg} \sqrt{\varepsilon}$ ensures that the zeros $|g| x=(1+\varepsilon)^{-1} e^{-i \chi}(-1 \pm i \sqrt{\varepsilon})$ of $V\left(|g| e^{i \chi}, \varepsilon\right)$ have nonvanishing imaginary part.

It is clearly enough to prove Proposition 2 for $H(|g|, \varepsilon,-\theta)$ and $H(|g|, \varepsilon, \chi)$ separately. We proceed by means of ODE techniques of WKB type because, while $H(|g|, \varepsilon,-\theta)$ can be analyzed by means of the Hunziker and Vock [8] stability theorem, this is not the case for $H(|g|, \varepsilon, \chi)$, because the union over $|g|>0$ of the numerical ranges is the whole of $\mathbb{C}$.

Lemma 3. Let $\varepsilon>0,|g| \geqq 0,0<\chi \leqq \eta$. Then the ODE $H(|g|, \varepsilon, \chi) \psi=0$, i.e.

$$
-\psi^{\prime \prime}+e^{-i(\pi-4 \eta)} x^{2}\left[\left(1+|g| e^{i \chi} x\right)^{2}+\varepsilon|g|^{2} e^{2 i \chi} x^{2}\right] \psi=0
$$

admits a unique solution $\psi_{-}(x,|g|, \varepsilon, \chi)$ (respectively $\psi_{+}(x,|g|, \varepsilon, \chi)$ ) which is $L^{2}$ at $-\infty$ (respectively at $+\infty)$, and such that

$$
\lim _{|g| \rightarrow 0} \psi_{ \pm}(x,|g|, \varepsilon, \chi)=\psi_{ \pm}(x, 0, \varepsilon, \chi) \equiv \psi_{ \pm}(x, 0, \chi)
$$

uniformly with respect to $(x, \chi) \in[-a, a] \times[\bar{\chi}, \eta], 0<a<+\infty, 0<\bar{\chi}<\eta$. An analogous statement holds for the solutions $\psi_{ \pm}(x,|g|, \varepsilon, \theta)$ of the $\mathrm{ODE} H(|g|, \varepsilon,-\theta) \psi=0$, $|\theta| \leqq \delta$, and for the solutions $\psi_{ \pm}(x,|g|, \varepsilon,-\chi)$ of the $\operatorname{ODE} H(|g|, \varepsilon,-\chi) \psi=0$, $-\eta \leqq \chi \leqq 0$.

Proof. We limit ourselves to consider the case of $\varepsilon>0$ suitably small, because this is clearly the most interesting and delicate situation. The general case requires only lengthier computations. For any $\varepsilon>0$ the function $V\left(|g| e^{i \chi}, \varepsilon\right)$ vanishes only at $x=0$ if $x \in \mathbb{R}$. Therefore we can define:

$$
f_{ \pm}(x,|g|, \varepsilon, \chi)=e^{i(\pi / 4-\eta)} V\left(|g| e^{i \chi}, \varepsilon\right)^{-1 / 4} \exp \left( \pm e^{-i \pi / 2+2 i \eta} \int_{0}^{x} V\left(|g| e^{i \chi}, \varepsilon\right)^{1 / 2} d t\right) .
$$

It is known (see e.g. Sibuya $\left[10\right.$, Lemma 13.1]) that $\psi_{ \pm}(\cdot)$ exist, with $\psi_{ \pm}(x,|g|, \varepsilon, \chi)$ $=(1+o(1)) f_{ \pm}(x,|g|, \varepsilon, \psi)$ as $x \rightarrow \pm \infty$, uniformly with respect to $(|g|, \chi)$, but this does not necessarily imply $f_{-}(x,|g|, \cdot) \rightarrow f_{-}(x, 0, \cdot)$. Consider now $f_{-}(\cdot)$. For $x<0$, setting $R(u, \varepsilon)=(1-u)^{2}+\varepsilon u^{2}$ we have:

$$
\int_{0}^{x} V\left(|g| e^{i \chi}, \varepsilon\right)^{1 / 2} d t=|g|^{-2} \int_{0}^{|g x|} t R\left(t e^{i \chi}, \varepsilon\right)^{1 / 2} d t=|g|^{-2} F(|g x|, \chi, \varepsilon),
$$


where

$$
\begin{aligned}
& F(|g x|, \chi, \varepsilon)=\frac{1}{3}(1+\varepsilon)^{-1} e^{-2 i \chi} R\left(|g x| e^{i \chi}, \varepsilon\right)^{3 / 2} \\
& \quad+\frac{1}{2}(1+\varepsilon)^{-2} e^{-2 i \chi}\left((1+\varepsilon) e^{i \chi}|g x|-1\right) R\left(|g x| e^{i \chi}, \varepsilon\right)^{1 / 2} \\
& \quad+\frac{1}{2} \varepsilon(1+\varepsilon)^{-3 / 2} e^{-2 i \chi} \ln \left[2(1+\varepsilon)^{1 / 2} e^{i \chi} R\left(|g x| e^{i \chi}, \varepsilon\right)^{1 / 2}+2|g x| e^{2 i \chi}-2 e^{i \chi}\right] \\
& \quad-\frac{1}{3}(1+\varepsilon)^{-1} e^{-2 i \chi}+\frac{1}{2} e^{-2 i \chi}(1+\varepsilon)^{-2}-\frac{1}{2} \varepsilon(1+\varepsilon)^{-3 / 2} e^{-2 i \chi} \ln \left(2(1+\varepsilon)^{1 / 2} e^{i \chi}-2 e^{i \chi}\right) .
\end{aligned}
$$

Set now $|g x|=1+y,-1 \leqq y \leqq+\infty$. For $\varepsilon$ and $\eta<\varepsilon$ suitably small, we can replace $e^{-i \pi / 2+2 i \eta}|g|^{-2} F(1+y, \chi, \varepsilon)$ through its first order Taylor expansion up to a relative error of order $\varepsilon^{2}$, which is uniform with respect to $y:$ namely, on account also of $\eta \geqq \chi$, we have:

$$
\begin{aligned}
& \operatorname{Im} e^{2 i \eta}|g|^{-2} F(1+y, \chi, \varepsilon) \geqq \operatorname{Im} e^{2 i \chi}|g|^{-2} F(1+y, \chi, \varepsilon) \\
& =\chi|g|^{-2} \frac{y^{2}(1+y)^{2}+\varepsilon\left(3 y^{4}+3 y+1+(1+y)\left(8 y^{2}+1 / 2\right)\right.}{(1+2 \varepsilon)\left(y^{2}+\varepsilon(1+y)^{2}\right)^{1 / 2}}\left(1+a(y ; \varepsilon, \chi) \varepsilon^{2}\right)
\end{aligned}
$$

for some $a(y ; \varepsilon, \chi)$ bounded independently of $(y ; \varepsilon, \chi)$. Hence

$$
\operatorname{Im}\left(e^{2 i \eta} \int_{0}^{x} V\left(|g| e^{i \chi}, \varepsilon\right)^{1 / 2} d t\right)>0
$$

strictly, independently of $x$ and $|g|$, as long as $\eta>0, \chi>0$, i.e. $\frac{\pi}{4} \geqq \theta \geqq \frac{\pi}{4}-\eta$. Therefore given $\bar{\varepsilon}>0$, there is $M(\bar{\varepsilon})<0$ independent of $|g|$ such that $\left|f_{-}(\cdot)\right|<\bar{\varepsilon}$ for $x<M(\bar{\varepsilon})$, and hence $\left|\psi_{-}(x,|g|, \chi, \varepsilon)\right|<\bar{\varepsilon}$ for $x<M(\bar{\varepsilon})$, uniformly with respect to $(|g|, \chi)$. This implies $\lim _{|g| \rightarrow 0} \psi_{-}(x,|g|, \chi, \varepsilon)=\psi_{-}(x, 0, \chi)$, with the stated uniformities by the theorem of the continuous dependence on the parameters applied to the ODE $H(|g|, \varepsilon, \chi) \psi=0$. For $\psi_{+}(x,|g|, \chi, \varepsilon)$ the statement is obvious because the real part of the integrand never undergoes a cancellation. An even simpler argument applies to $\psi_{ \pm}(x,|g|, \theta, \varepsilon)$ : in this case indeed one has to consider the real part of

$$
\pm|g|^{-2} e^{-2 i \theta} \int_{0}^{|g| x} t R(-|g| t, \varepsilon)^{1 / 2} d t
$$

which for $|\theta|<\frac{\pi}{4}$ is trivially uniformly positive as $x \rightarrow \pm \infty$, respectively. This proves Lemma 3, and explains why a different scaling is needed for $\theta= \pm \pi / 4$.

Remark. The above statement is not true for $\varepsilon=0$. Taking indeed $\theta=0$, the double zero of $V(g, 0)=x^{2}(1+g x)^{2}$ at $x=-\frac{1}{g}$ forces the exponent of the WKB solution to
switch sign near $-\infty$ as $g \rightarrow 0$.

Lemma 4. Let $R(|g|, \varepsilon, \chi ; z)=(H(|g|, \varepsilon, \chi)-z)^{-1}, \quad R(|g|, \varepsilon, \theta, z)=(H(|g|, \varepsilon,-\theta)-z)^{-1}$ denote the resolvent, of $H(|g|, \varepsilon, \chi)$ and $H(|g|, \varepsilon,-\theta)$, respectively, which are compact operators in $L^{2}$ by Proposition 1 for $z \notin \sigma(H(\cdot))$. Let $R(0, \theta, z)=\left(p^{2}+e^{-4 i \theta} x^{2}-z\right)^{-1}$, $R(0, \eta, z)=\left(p^{2}+e^{-i \pi+4 i \eta} x^{2}-z\right)^{-1}$ denote the unperturbed scaled resolvents, $z \neq e^{-2 i \theta}(2 n+1), z \neq e^{-i \pi / 2+2 i \eta}(2 n+1), n=0,1, \ldots$, respectively. Then, as $|g| \rightarrow 0$, and $z \neq e^{-2 i \theta}(2 n+1), \quad z \neq e^{-i \pi / 2+2 i \eta}(2 n+1)$, respectively, $\|R(|g|, \varepsilon, \theta, z)-R(0, \theta, z)\| \rightarrow 0$, 
$\|R(|g|, \varepsilon, \chi, z)-R(0, \eta, z)\| \rightarrow 0$ the convergence being uniform with respect to $\theta,|\theta| \leqq \frac{\pi}{4}-\delta$, and $\chi, 0<\chi \leqq \eta$, respectively.

Proof. Denote by $W_{\theta}(|g|, \varepsilon), W_{\chi}(|g|, \varepsilon)$ the Wronskians of $\left(\psi_{-}(x,|g|, \varepsilon, \theta)\right.$. $\left.\psi_{+}(x,|g|, \varepsilon, \theta)\right)$ and $\left(\psi_{-}(x,|g|, \varepsilon, \chi), \psi_{+}(x,|g|, \varepsilon, \chi)\right)$, respectively, and by $W_{\theta}(0), W_{\eta}(0)$ the Wronskians of $\left(\psi_{-}(x, 0, \theta), \psi_{+}(x, 0, \theta)\right),\left(\psi_{-}(x, 0, \eta), \psi_{+}(x, 0, \eta)\right)$, respectively. Since $W_{\theta}(0) \neq 0, W_{\eta}(0) \neq 0$, by Lemma 3 there is $\bar{g}>0$ independent of $\theta, \chi$, respectively, such that $W_{\theta}(|g|, \varepsilon) \neq 0, W_{\chi}(|g|, \varepsilon) \neq 0$ for $|g| \leqq \bar{g}$. Then, through standard ODE arguments (see e.g. Hellwig [4]), one can easily check the following Green's function representations, valid for any $u \in L^{2}$

$$
\begin{aligned}
(R(|g|, \varepsilon, \theta ; 0) u)(x) & =\int_{\mathbb{R}} G(x, y ;|g|, \varepsilon, \theta) u(y) d y, \\
(R(|g|, \varepsilon, \chi ; 0) u)(x) & =\int_{\mathbb{R}} G(x, y ;|g|, \varepsilon, \chi) u(y) d y, \\
(R(0, \theta ; 0) u)(x) & =\int G(x, y ; 0, \theta) u(y) d y, \\
(R(0, \eta ; 0) u)(x) & =\int_{\mathbb{R}} G(x, y ; 0, \eta) u(y) d y,
\end{aligned}
$$

where

$$
\begin{aligned}
& G(x, y ;|g|, \varepsilon, \theta)=W_{\theta}(|g|, \varepsilon)^{-1} \begin{cases}\psi_{-}(y, \cdot) \psi_{+}(x, \cdot), & y \leqq x \\
\psi_{+}(y, \cdot) \psi_{-}(x, \cdot), & y \geqq x,\end{cases} \\
& G(x, y ;|g|, \varepsilon, \chi)=W_{\chi}(|g|, \varepsilon)^{-1} \begin{cases}\psi_{-}(y, \cdot) \psi_{+}(x, \cdot), & y \leqq x \\
\psi_{+}(y, \cdot) \psi_{-}(x, \cdot), & y \geqq x,\end{cases}
\end{aligned}
$$

and analogous definitions for $G(x, y ; 0, \theta), G(x, y ; 0, \eta)$, with $W_{\theta}(|g|, \varepsilon), W_{\chi}(|g|, \varepsilon)$ replaced by $W_{\theta}(0), W_{\eta}(0)$, respectively. Starting from the asymptotic behaviours of $\psi_{ \pm}(\cdot)$, it is not difficult to check that $G(x, y ;|g|, \varepsilon, \chi)$ and $G(x, y ;|g|, \varepsilon, \theta)$ are HilbertSchmidt integral kernels, as well as $G(\cdot, 0, \eta)$ and $G(\cdot, 0, \theta)$. Proceeding as in Lemma 3, one easily proves that, given $\bar{\varepsilon}>0$, there is $M(\bar{\varepsilon})>0$ independent of $|g|$ and $\theta, \chi$ (respectively) such that

$$
\begin{array}{r}
\iint_{x^{2}+y^{2} \geqq M(\bar{\varepsilon})}|G(x, y ;|g|, \varepsilon, \theta)|^{2} d x d y<\bar{\varepsilon}, \\
\quad \iint_{x^{2}+y^{2} \geqq M(\bar{\varepsilon})}|G(x, y ;|g|, \varepsilon, \chi)|^{2} d x d y<\bar{\varepsilon} .
\end{array}
$$

Therefore, by the continuity of $\psi_{ \pm}(x,|g|, \varepsilon, \theta), \psi_{ \pm}(x,|g|, \varepsilon, \chi)$ as $|g| \rightarrow 0$, uniform with respect to $\theta$ and $\chi$, respectively, and with respect to $x$ in the compacts of $\mathbb{R}$, and by the uniform convergence of $W_{\theta}(|g|, \varepsilon), W_{\chi}(|g|, \varepsilon)$ towards $W_{\theta}(0)$ and $W_{\eta}(0)$, respectively, we have for $|g| \rightarrow 0$,

$$
\begin{aligned}
& \int_{\mathbb{R}^{2}}|G(x, y ;|g|, \varepsilon, \theta)-G(x, y, 0, \theta)|^{2} d x d y \rightarrow 0, \\
& \int_{\mathbb{R}^{2}}|G(x, y ;|g|, \varepsilon, \chi)-G(x, y, 0, \eta)|^{2} d x d y \rightarrow 0 .
\end{aligned}
$$


This is enough to prove the assertion because the Hilbert-Schmidt norm majorizes the operator norm, and the norm resolvent convergence for $z=0$ implies the norm resolvent convergence for all $z$ as above. This proves Lemma 4 .

Proof of Proposition 2. Let $E=(2 j+1) ; j=0,1, \ldots$ be an unperturbed eigenvalue, which is an eigenvalue also of $e^{-2 i \phi} H_{0}(\phi)=e^{-2 i \phi}\left(p^{2}+e^{+4 i \phi} x^{2}\right),|\phi|<\frac{\pi}{4}$. By Lemma 4 and standard arguments of perturbation theory (see e.g. Reed and Simon [13, Sect. XII.3), $E$ is stable both as an eigenvalue of $e^{2 i \theta} H(|g|, \varepsilon,-\theta)$, $|\theta| \leqq \frac{\pi}{4}-\delta$, and as an eigenvalue of $e^{-i(\pi / 2-2 \eta)} H(|g|, \varepsilon, \pm \chi), 0 \leqq \chi \leqq \eta$. This implies that given the circle $\Gamma_{v}:\{z:|z-E|=v\}$ there is $B(E)>0$ such that $e^{2 i \theta} H(|g|, \varepsilon,-\theta)$ and $e^{-i(\pi / 2-2 \eta)} H(|g|, \varepsilon, \pm \chi)$ have one and only one eigenvalue, denoted by $E(|g|, \theta)$ and $E(|g| ; \pm \chi)$, respectively, inside $\Gamma_{v}$ for all $g$ such that $|g| \leqq B(E)$, with

$$
\lim _{|g| \rightarrow 0} E(|g|, \theta)=\lim _{|g| \rightarrow 0} E(|g|, \chi)=E .
$$

By rescaling the phase of $g$ we can thus conclude that $H(g, \varepsilon)$ has one and only one eigenvalue $E(g, \varepsilon)$ inside $\Gamma_{v}$ as long as $|g|<B(E),|\theta|=|\arg g| \leqq \frac{\pi}{4}$, with $E(g, \varepsilon) \rightarrow E$ as $g \rightarrow 0$. In addition $E(g, \varepsilon)$ is a holomorphic function of $g$ at least for $0<|g|<B(E)$, $|\arg g| \leqq \frac{\pi}{4}$. This proves assertions (i) and (ii). To see (iii), by the scaling invariance of the RSPE and well known arguments of asymptotic perturbation theory (see e.g. Reed and Simon [13, Sect. XII.4]) it is enough to check that both $R(|g|, \varepsilon, \chi, z)$ and $R(|g|, \varepsilon, \theta, z)$ are uniformly bounded with respect to $(|g|, z) \in(0, B(E)) \times \Gamma_{1 / 2}$. This is once more a consequence of the norm resolvent convergence.

Remark. The limit $\varepsilon \rightarrow 0$ of $H(g, \varepsilon)$ is of course highly irregular. Examined in the light of the stability of the boundary conditions, for $g$ real it would clearly correspond to a fixed choice of sign in $\sqrt{(1+g x)^{2}+\varepsilon g^{2} x^{2}}$ at the limit $\varepsilon=0$ also for $x=-\frac{1}{g}$ while the square root is forced to switch sign at the double zero $x=-\frac{1}{g}$ by analyticity. Not surprisingly, the Borel sum of the RSPE of $H(g)$ does not represent an eigenvalue of the problem and has been identified [3] as a complex eigenvalue of a non-self-adjoint problem described by the same equation with $L^{2}$ conditions at infinity imposed along complex directions.

Acknowledgements. We thank Professors B. Simon and T. Spencer for suggesting the problem and their interest in this work.

\section{References}

1. Coleman, S.: The uses of instantons. Proceedings Intern. School of Physics, Erice 1977

2. Gildener, E., Patrasciou, A.: Pseudoparticle contributions to the energy spectrum of a onedimensional system. Phys. Rev. D16, 423-433 (1977)

3. Graffi, S., Grecchi, V.: The Borel sum of the double-well perturbation series and the Zinn-Justin conjecture. Phys. Lett. 121 B, 410 (1983) 
4. Hellwig, G.: Differentialoperatoren der mathematischen Physik. Berlin, Heidelberg, New York: Springer 1964

5. Herbst, I., Simon, B.: Some remarkable examples in eigenvalue perturbation theory. Phys. Lett. 78B, 304 (1978)

6. 't Hooft, G.: Borel summability of a four-dimensional field theory. Phys. Lett. 119B, 369 (1982)

7. 't Hooft, G.: Is asymptotic freedom enough? Phys. Lett. 109B, 474 (1982)

8. Hunziker, W., Vock, E.: Stability of Schrödinger eigenvalue problems. Commun. Math. Phys. 83, $208(1982)$

9. Kato, T.: Perturbation theory for linear operators. Berlin, Heidelberg, New York: Springer 1966

10. Sibuya, Y.: Global theory of a second order differential equation with a polynomial coefficient. Amsterdam: North-Holland 1975

11. Simon, B.: Instantons, double-wells, and large deviations. California Institute of Technology (preprint)

12. Simon, B.: Coupling constant analyticity for the anharmonic oscillator. Ann. Phys. 58, 76 (1970)

13. Reed, M., Simon, B.: Methods of modern mathematical physics. IV. Analysis of operators. New York: Academic Press 1978

14. Sokal, A.: An improvement of Watson's theorem on Borel summability. J. Math. Phys. 21, 261 (1980)

Communicated by B. Simon

Received April 25, 1983; in revised form September 12, 1983 
